\title{
COVID-19 em pediatria: sugestões para o manejo nutricional
}

COVID-19 in pediatrics: suggestions for nutritional management

DOI: $10.37111 /$ braspeni.AE202035201

Bianca Stachissini Manzoli

Patricia Zamberlan'

Analisa Gabriela Zuchi Leite'

Ana Paula Alves Reis ${ }^{1}$

\section{Unitermos:}

Betacoronavirus. Infecções por Coronavirus. Pediatria. Abastecimento de Alimentos. Terapia nutricional.

\section{Keywords:}

Betacoronavirus. Coronavirus Infections. Pediatrics. Food Supply. Nutrition therapy.

\section{Endereço de correspondência:}

Bianca Stachissini Manzoli

Avenida Rouxinol, 837 - apto $32 \mathrm{~A}$ - Moema - São

Paulo, SP, Brasil - CEP 04516-001

E-mail: bianca.manzoli@hc.fm.usp.br

\section{Submissão}

19 de maio de 2020

Aceito para publicação

22 de junho de 2020

\section{RESUMO}

Poucos são os dados que demonstram o impacto da doença provocada pelo vírus SARS-CoV-2 (batizada de COVID-19) na população pediátrica. No entanto, é sabido que crianças, especialmente aquelas de mais baixa idade, constituem um grupo suscetível a infecções, devido a algumas particularidades fisiológicas, como imaturidade do sistema imunológico, e, portanto, necessitam de especial atenção durante a pandemia. Recém-nascidos, lactentes, crianças e adolescentes internados com suspeita ou confirmação da COVID-19 devem receber assistência nutricional adequada. Com base nas informações do pequeno número de casos pediátricos de COVID-19 (frente à população adulta), no conhecimento das características fisiológicas desta faixa etária e na compreensão epidemiológica da SARS-CoV-2, sugestões de abordagem prática para atendimento destes pacientes em unidades hospitalares foram elaboradas.

\section{ABSTRACT}

Few data show the impact of the disease caused by the SARS-CoV-2 virus (called COVID-19) on the pediatric population. However, it is known that children, especially those of a younger age, are a group susceptible to infections, due to some particularities such as immaturity of the immune system, and therefore need special attention during the pandemic. Newborns, infants, children and adolescents hospitalized with suspected or confirmed COVID-19 should receive adequate nutritional assistance. Based on information from the small number of pediatric cases of COVID-19 (compared to the adult population), on the knowledge of the physiological characteristics of this age group and on the epidemiological understanding of SARS-CoV-2, suggestions for a practical approach to care for these patients in units hospitals were developed.

1. Nutricionista do Instituto da Criança e do Adolescente, Hospital das Clínicas, Faculdade de Medicina, Universidade de São Paulo, São Paulo, SP, Brasil. 


\section{INTRODUÇÃO}

No início de dezembro de 2019, a doença causada pelo vírus SARS-CoV-2 (batizada de COVID-19) emergiu na cidade chinesa de Wuhan e, desde então, se disseminou rapidamente em todo o mundo, transformando-se em uma pandemia ${ }^{1}$. A doença clínica, também chamada síndrome respiratória aguda grave-coronavírus-2 (SARS-CoV-2), provavelmente é originária de morcegos, e apresenta um curso clínico ainda pouco conhecido, especialmente na faixa etária pediátrica².

Estudos disponíveis até o momento relatam que a população pediátrica representa cerca de $1 \%$ a $5 \%$ dos casos diagnosticados com COVID-19. A maioria dos pacientes pediátricos (indivíduos com idade inferior a 18 anos) infectados tem apresentado evolução clínica menos grave que a dos adultos; estimativas indicam que, na maior série de casos infantis registrada até agora, cerca de $90 \%$ das crianças com o diagnóstico de COVID-19 apresentam doença assintomática, leve ou moderada ${ }^{3}$.

Enquanto em adultos as manifestações respiratórias graves são comuns, um estudo realizado no hospital infantil de Wuhan demonstrou que, na pediatria, os pacientes apresentaram manifestações clínicas típicas de infecções respiratórias agudas, como tosse $(48,5 \%)$, eritema faríngeo $(46,2 \%)$ e febre de pelo menos $37,5^{\circ} \mathrm{C}$ $(41,5 \%)$; além de diarreia $(8,8 \%)$, fadiga $(7,6 \%)$, rinorreia $(7,6 \%)$ e vômitos $(6,4 \%)$. Em apenas quatro de 171 crianças $(2,3 \%)$, a saturação de oxigênio mostrou-se inferior a $92 \%{ }^{4-6}$.

Lactentes e crianças são tipicamente de alto risco para admissão no hospital por infecções virais do trato respiratório, porém pouco se sabe sobre as características clínicas e epidemiológicas de pacientes pediátricos com COVID-19. Em condições de infecções, o estado nutricional, por vezes, é capaz de predizer desfechos clínicos, e, portanto, torna-se um aspecto importante a ser considerado na avaliação e no manejo destas crianças durante a pandemia.

Com base nas informações disponibilizadas pela Organização Mundial da Saúde (OMS), esta abordagem tem como objetivo padronizar as principais condutas nutricionais a serem realizadas na avaliação e no manejo de pacientes pediátricos acometidos pelo vírus ${ }^{7}$. É importante salientar que tais sugestões derivam-se do conhecimento existente sobre a assistência nutricional prestada à população pediátrica hospitalizada, uma vez que, até o momento, não existem recomendações específicas para a doença (infecção por COVID-19).

\section{ASSISTENCIA NUTRICIONAL AO PACIENTE HOSPITALIZADO}

\section{Avaliação Nutricional}

Os pacientes pediátricos suspeitos ou com confirmação da COVID-19 internados devem ser monitorados pela equipe de nutrição, sendo proposto e implementado um plano de cuidado nutricional.

A triagem nutricional deve ser realizada nas primeiras 24 horas de admissão do paciente, com o objetivo de identificar o risco que a criança ou adolescente apresenta para subnutrir durante a internação e, assim, instituir uma intervenção nutricional precoce, se necessária.

De modo geral, a triagem nutricional é realizada pelo nutricionista, cuja entrevista com o responsável deve ser concretizada, preferencialmente, à distância. No caso da entrevista presencial, recomendam-se os equipamentos de proteção individual (EPIs) estabelecidos por protocolos institucionais, e que devem seguir as recomendações da Agência Nacional de Vigilância Sanitária (ANVISA) do Ministério da Saúde (MS). Entretanto, a depender do protocolo institucional, qualquer outro profissional, desde que devidamente treinado, pode aplicar o questionário com o responsável enfermeiro, técnico de enfermagem, médico, fisioterapeuta, farmacêutico. Várias são as ferramentas disponíveis para a triagem nutricional de crianças. Nós temos sugerido a StrongKids ${ }^{8}$, por ser rápida, de fácil aplicação, e sem a necessidade de incorporar dados objetivos ${ }^{7,9}$.

A avaliação pode incluir dados de peso, estatura ou comprimento, circunferência do braço e perímetro cefálico para crianças até dois anos de idade. No entanto, em virtude da atual situação, o uso de tais parâmetros nem sempre é possível. A inclusão desses dados em protocolos deve ser avaliada com cautela, até mesmo para considerar o diagnóstico encontrado frente a outras ferramentas utilizadas.

Logo, a classificação do estado nutricional deve ser obtida a partir de peso e estatura aferidos pela equipe de enfermagem, ou referidos pela mãe ou acompanhante responsável.

A anamnese alimentar ou visitas diárias podem ser feitas de forma presencial, desde que o nutricionista esteja devidamente paramentado (máscara N95, óculos de proteção, avental e luvas) ou, preferencialmente, por telefone, se houver esta possibilidade.

Com base no risco para subnutrição, diagnóstico nutricional e estimativa das necessidades nutricionais (que variam de acordo com idade, sexo, peso e estatura), a conduta dietoterápica deve ser instituída. A reavaliação deve ser realizada semanalmente, ou de acordo com o protocolo da instituição. A cada reavaliação, sempre que houver necessidade, o plano de cuidado nutricional deverá ser redefinido. 


\section{Acompanhamento Nutricional por Nível de Assistência ${ }^{10,11}$}

- Nível primário - quando a criança apresenta baixo risco nutricional (mensurado pelo StrongKids ${ }^{8}$ ) e nenhuma dietoterapia específica. A avaliação antropométrica deve ser realizada, se necessário, e repetida a cada 15 dias. A triagem deve ser refeita semanalmente.

- Nível secundário - quando a criança apresenta médio ou alto risco nutricional (mensurado pelo StrongKids ${ }^{8}$ ) ou alguma dietoterapia específica. Deve ser realizada visita diária ao paciente, avaliação antropométrica inicial em 72 horas e repetida a cada 10 dias.

- Nível terciário - quando a criança apresenta médio ou alto risco nutricional (mensurado pelo StrongKids ${ }^{8}$ ) e alguma dietoterapia específica. Deve ser realizada visita diária ao paciente, avaliação antropométrica inicial em 72 horas e repetida a cada 7 dias.

\section{Cálculo das Necessidades Nutricionais}

O cálculo das necessidades nutricionais sempre deve ser precedido de uma avaliação nutricional e levar em conta a doença de base, bem como o estado metabólico e clínico atual do paciente.

O padrão-ouro para a determinação das necessidades de energia é a calorimetria indireta, entretanto, recomenda-se a não realização do procedimento nestes pacientes, pelo risco de disseminação do vírus, especialmente, se não houver as medidas de segurança exigidas. Na ausência desse método, existem diversas equações para a sua estimativa. A equação recomendada para a estimativa das necessidades calóricas de crianças e adolescentes gravemente doentes internadas por COVID-19 é a de Schofield'2 (Quadro 1).

O cálculo das necessidades nutricionais de pacientes com excesso de peso deve considerar o peso ideal e não o peso atual ou habitual. Para menores de dois anos, o peso ideal é aquele correspondente ao escore-z zero de peso para idade $(P / I)$, do referencial da OMS/2006$2007^{13}$. O peso ideal de maiores de dois anos é obtido a partir do índice de massa corpórea (IMC) ideal para idade, ou seja, aquele cujo escore-z é igual a zero, sendo o cálculo realizado considerando a altura atual da criança. No caso de pacientes com magreza, segundo IMC para idade (IMC/I), pode-se considerar um acréscimo calórico visando à recuperação do estado nutricional. Entretanto, é importante evitar excessos devido ao risco de síndrome de realimentação.

Pacientes gravemente doentes, de todas as idades, devem receber oferta proteica mínima de 1,5 g de proteína $/ \mathrm{kg} / \mathrm{dia}^{14}$, a fim de evitar balanço nitrogenado negativo.
Quadro 1 - Equação para estimar o requerimento energético de crianças e adolescentes gravemente doentes (Schofield, 1985) 12 .

\begin{tabular}{|c|c|c|}
\hline Idade (anos) & Sexo & Equação \\
\hline \multirow[t]{2}{*}{$0-3$} & Masculino & $\begin{array}{l}\text { TMB }=(59,48 \times P)-30,33 \\
T M B=(0,167 \times P)+(1517,4 \times E)-617,6\end{array}$ \\
\hline & Feminino & $\begin{array}{l}\mathrm{TMB}=(58,29 \times \mathrm{x})-31,05 \\
\mathrm{TMB}=(16,25 \times \mathrm{x})+(1023,2 \times E)-413,5\end{array}$ \\
\hline \multirow[t]{2}{*}{$3-10$} & Masculino & $\begin{array}{l}\mathrm{TMB}=(22,7 \times P)+505 \\
\mathrm{TMB}=(19,6 \mathrm{xP})+(130,3 \times \mathrm{E})+414,9\end{array}$ \\
\hline & Feminino & $\begin{array}{l}\text { TMB }=(20,3 \times P)+486 \\
T M B=(16,97 \times P)+(161,8 \times E)+371,2\end{array}$ \\
\hline \multirow[t]{2}{*}{$10-18$} & Masculino & $\begin{array}{l}\text { TMB }=(13,4 \times P)+693 \\
\text { TMB }=(16,25 \times P)+(137,2 \times E)+515,5\end{array}$ \\
\hline & Feminino & $\begin{array}{l}\text { TMB }=(17,7 \times P)+659 \\
T M B=(8,365 \times P)+(465 \times E)+200\end{array}$ \\
\hline
\end{tabular}

Onde: $\mathrm{P}=$ peso $(\mathrm{kg}) ; \mathrm{E}=$ estatura $(\mathrm{m})$

\section{Terapia Nutricional}

A COVID-19 causa uma série de sintomas que prejudicam a aceitação alimentar, como falta de ar, náuseas e vômitos, disgeusia e anosmia, além de febre, que pode elevar as demandas de energia. Sendo assim, frequentemente, a terapia nutricional (TN) se fará necessária, devendo ser instituída com base em protocolos institucionais (Figura 1) 15, que devem seguir as boas práticas da terapia nutricional enteral (TNE) e parenteral (TNP) ${ }^{16,17}$, levando em conta o risco nutricional e a avaliação da aceitação alimentar do paciente frente às suas demandas nutricionais.

Como não há dados específicos do manejo nutricional para pacientes infectados por COVID-19, atualmente, as considerações podem pautar-se no conhecimento já existente sobre a TN em outras condições, bem como na experiência clínica.

Recente publicação da Society of Critical Care Medicine e American Society for Parenteral and Enteral Nutrition (ASPEN) ${ }^{18}$ pontua alguns aspectos importantes da TN em pacientes com COVID-19 internados em unidade de terapia intensiva, e que podem ser extrapolados para a pediatria:

1) Tempo de início da $\mathrm{TN}$ - um dos aspectos mais importantes do manejo nutricional destes pacientes é quando iniciar a TN. Recomenda-se iniciar a nutrição enteral (NE) de forma precoce nas primeiras 24 a 36 horas após a admissão na UTI ou 12 horas após a intubação, até mesmo em pacientes com choque circulatório que, via de regra, parecem tolerar bem a NE trófica; 


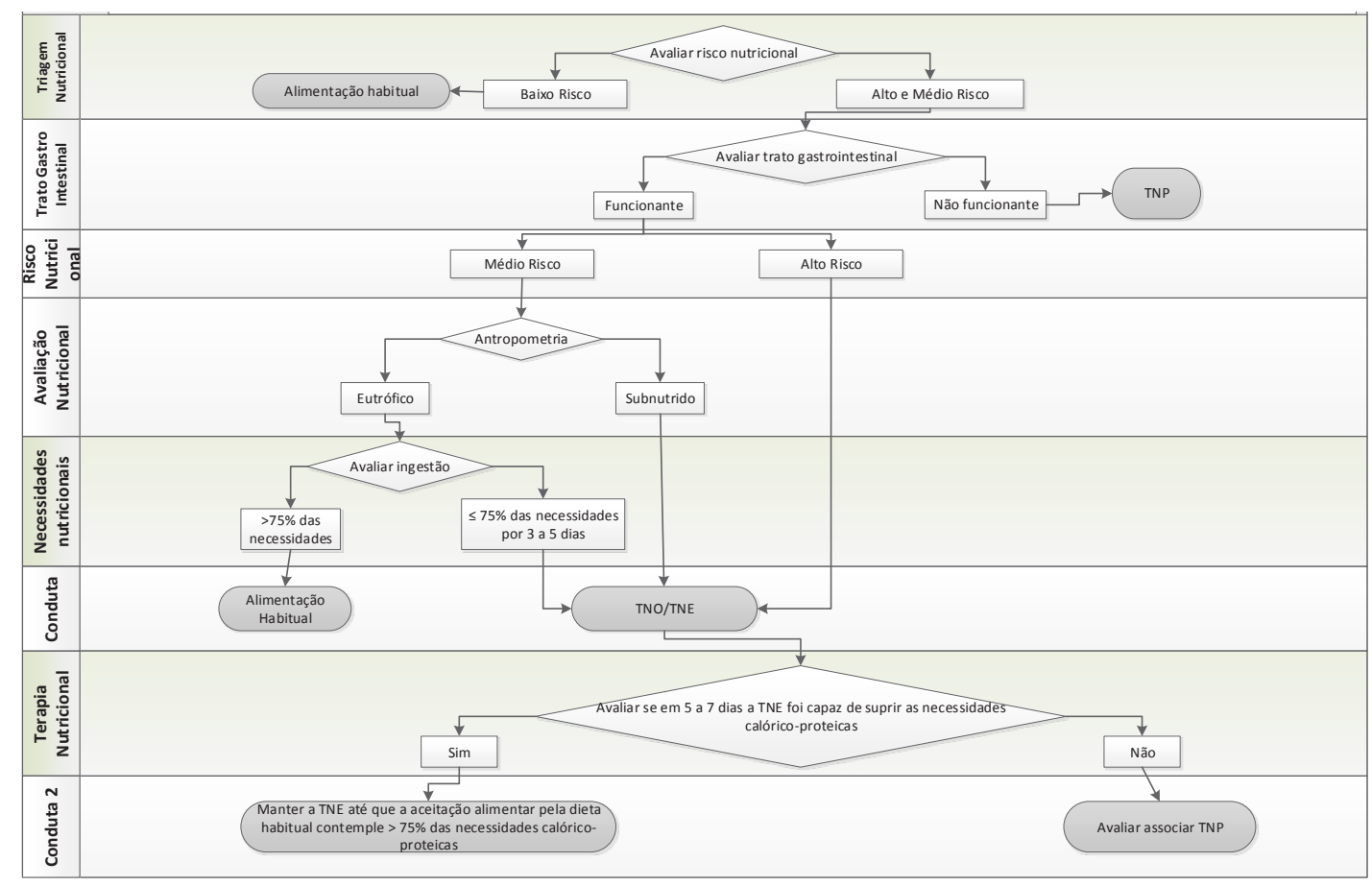

Figura 1 - Algoritmo de indicação de terapia nutricional em pediatria ${ }^{13}$.

TNE: terapia nutricional; TNO: terapia nutricional oral; TNP: terapia nutricional parenteral

2) Via de administração - A NE é a via preferida à NP para a oferta de nutrientes aos pacientes. Recomenda-se o uso da sonda em posição gástrica (utilizada rotineiramente em pediatria) e, nos casos de intolerância, a introdução de um agente pró-cinético. A sonda na posição pós-pilórica deve ser utilizada somente quando houver falha da conduta anterior, para minimizar a violação do isolamento aéreo e limitar a exposição dos profissionais de saúde. Isto porque a colocação de qualquer dispositivo de acesso entérico pode provocar tosse, devendo ser considerado um procedimento gerador de aerossol (manter a boca do paciente coberta durante a colocação nas narinas). Os pacientes que necessitam da sonda pós-pilórica devem ser submetidos à colocação no leito com técnicas que não requerem uso de endoscopia. Quanto à infusão da dieta, recomenda-se, fortemente, a modalidade contínua em detrimento da intermitente para reduzir o contato do profissional com o paciente infectado, e consequente exposição ao vírus.

Antes do aparecimento dos sintomas respiratórios, alguns pacientes apresentam inicialmente sintomas gastrointestinais, como diarreia, náusea, vômito, desconforto abdominal e, em alguns casos, sangramento; sendo que várias evidências sugerem que o desenvolvimento destes sintomas indica maior gravidade da doença. Para estes pacientes, e para aqueles que apresentam intolerância à $\mathrm{NE}$, a introdução da NP está indicada. A transição para
NE é sugerida com a melhora ou o desaparecimento dos sintomas gastrointestinais;

3) Progressão e monitorização da TN - a TN, especialmente a NE, deve ser iniciada em pequenos volumes, com progressão lenta, de modo a atingir 70\% das necessidades nutricionais na primeira semana. Recomenda-se uma quantidade mínima de 1,5 g de proteína $/ \mathrm{kg} /$ dia para evitar balanço nitrogenado negativo. As dietas "standard" devem ser a primeira opção de escolha, sem nenhuma evidência para o uso rotineiro das chamadas dietas imunomoduladoras. Não é recomendada a avaliação do resíduo gástrico para avaliação da tolerância à $\mathrm{NE}$, a fim de reduzir o risco de transmissão do COVID-19 ao profissional de saúde.

\section{Aleitamento Materno}

Em casos de mulheres infectadas que amamentam, tem sido orientado que a amamentação deve ser mantida, uma vez que não há elementos que comprovem que o vírus possa ser transmitido ao lactente pelo leite materno. Uma revisão publicada pelo Centers for Disease Control and Prevention $(C D C)^{19}$ norte-americano e outra pelo Royal College of Obstetricians and Gynaecologists $(\text { RCOG })^{20}$ de Londres afirmam que "os benefícios da amamentação ainda superam quaisquer riscos potenciais de transmissão do vírus através do leite materno". No 
ambiente e prática hospitalar, o processo, por vezes, torna-se inviável, em virtude de um risco mais elevado do recém-nascido (RN) ou lactente infectar-se ${ }^{21}$. No caso de alojamento conjunto, é possível manter a amamentação, desde que realizada em quarto privativo com precaução de contato para gotículas, procurando manter distanciamento de 2 metros entre o leito materno e o berço do $\mathrm{RN}$, além da restrição de visita ${ }^{21}$.

Na possibilidade da mãe infectada amamentar (em casa ou alojamento conjunto, por exemplo) ou quando o RN é positivo para COVID-19, mas a mãe está saudável, o processo deve seguir as "recomendações e medidas preventivas adequadas"22:

- A amamentação deve ser mantida desde que a mãe deseje amamentar e esteja em condições clínicas adequadas para fazê-lo;

- A mãe deve ser orientada a aderir às medidas apresentadas a seguir, com o propósito de reduzir o risco de transmissão do vírus (da mãe para o lactente e viceversa) por gotículas respiratórias durante o contato com a criança, incluindo a amamentação;

- Lavar as mãos por, pelo menos, 20 segundos antes de tocar a criança, ou antes de ordenhar o leite materno;

- Usar máscara facial (cobrindo completamente nariz e boca) durante as mamadas, e evitar falar ou tossir durante a amamentação;

- Trocar a máscara imediatamente em caso de tosse ou espirro, ou a cada nova mamada;

- Em caso de opção pela extração do leite, é recomendada, preferencialmente, a extração manual. Entretanto, se for utilizada uma bomba extratora, devem ser observadas as seguintes orientações para a higienização:

- Primeiramente remover os resíduos das peças da bomba extratora (válvulas, funis e frascos) com água potável;

- Em seguida, lavar as peças com água morna (aproximadamente a $30^{\circ} \mathrm{C}$ ) e detergente líquido;

- Enxaguar as peças novamente em água corrente por 15 a 20 segundos;

- Realizar a desinfecção das peças com água fervente. Como são vários os modelos e marcas de bomba extratora, o método de desinfecção das peças deve seguir o recomendado pelo fabricante do equipamento;

- Secar as peças com um pano limpo e colocá-las de cabeça para baixo, deixando a bomba extratora pronta para a próxima ordenha;

- Com relação ao armazenamento do leite ordenhado, devem-se seguir as recomendações disponíveis no documento "Cartilha para a mãe trabalhadora que amamenta" ${ }^{23}$;
- Considerar a possibilidade de solicitar a ajuda de alguém que esteja saudável (não infectado), treinado por um profissional de saúde, para oferecer o leite materno ao lactente em copinho, xícara ou colher.

\section{Lactário}

Idealmente, o lactário tem o objetivo de fornecer alimentação apropriada e segura, do ponto de vista microbiológico e nutricional, garantindo a promoção da saúde e/ou recuperação dos RNs, lactentes e crianças ${ }^{24}$.

Em algumas situações especiais, quando a manipulação não atende às condições higiênico-sanitárias adequadas, esses processos podem ser o foco de transmissão de microrganismos patogênicos, sendo fundamental a utilização de medidas que minimizem os riscos, bem como a implementação de fluxos de atividades que evitem a contaminação cruzada ${ }^{24,25}$.

A higienização é qualquer procedimento que elimina ou reduz os perigos microbiológicos até níveis suportáveis, minimizando os riscos de transmissão de agentes patogênicos causadores de doenças. A higienização compreende desde a limpeza, passando por lavagem, desinfecção e chegando até a esterilização ${ }^{24}$, a saber:

- Limpeza: procedimento que envolve a simples remoção de sujidades ou resíduos macroscópicos;

- Lavagem: procedimento que envolve a utilização de água e sabão líquido ou detergente, para melhor remoção das sujidades, podendo ou não reduzir os patógenos a níveis suportáveis;

- Desinfecção: operação de redução, por métodos físicos e/ ou químicos, do número de microrganismos patogênicos a níveis aceitáveis;

- Esterilização: qualquer processo físico ou químico que elimine todas as formas de vida microbiana.

A limpeza corresponde à etapa mais importante do processo de higienização, e a desinfecção visa à destruição de micro-organismos que causam doenças (como vírus, bactérias e fungos), mediante a aplicação de agentes físicos e/ou químicos.

Para a higienização de utensílios utilizados por pacientes infectados, como mamadeiras no caso de crianças, é recomendado 24,26 :

- Seguir as recomendações da CCHI da instituição;

- Que as mamadeiras sejam recolhidas pela equipe de enfermagem em recipientes exclusivos e entregues para o copeiro, que, então, as encaminhará para a área de higienização exclusiva para este fim; as sujidades sejam removidas, utilizando enxágue em água corrente; o recipiente utilizado para o recolhimento seja higienizado e desinfectado; que os produtos para o processo de desinfecção possuam registro 
junto à legislação vigente e sejam avaliados com relação à sua eficácia, além de validados e aprovados pela $\mathrm{CCIH}$ ou Comissão de Vigilância Sanitária (CVS);

- Após o processo de desinfecção, as mamadeiras devem ser encaminhadas para o lactário;

- No lactário, as mamadeiras e acessórios passam pelo processo de lavagem com água e detergente próprio, escovas exclusivas e enxague, que pode ser manual ou mecânico;

- Após utilização da escova de higienização, estas devem ser desinfetadas com solução de hipoclorito a 0,02 ppm por 1 hora, deixar escorrer a solução e secar naturalmente (após secagem, armazenar em saco plástico identificado para este uso exclusivo);

- Imediatamente após, as mamadeiras devem ser levadas para a desinfecção física de alto nível ou esterilização em autoclave. Para o método de desinfecção química (médio nível), recomenda-se preferencialmente o uso de hipoclorito de sódio a $200 \mathrm{ppm}$ ou $0,02 \%$ de cloro ativo para superfícies, utensílios e equipamentos de contato com alimentos, com tempo mínimo de exposição à solução de 15 minutos. Não utilizar diluição inferior a 100 ppm ou superior a 250 ppm;

- Como meio de segurança nos processos, é valido ressaltar a importância de uso de EPIs (luvas, óculos, avental, máscara) nos processos de limpeza e desinfecção, bem como na esterilização, e realizar a desparamentação de forma correta, garantindo, assim, a integridade física e a saúde do funcionário;

- Nos casos em que houver necessidade do lactarista entrar no quarto do paciente infectado, este deve utilizar os 4 EPIs (máscara N95, óculos de proteção, avental e luvas).

\section{Banco de Leite Humano (BLH)}

- Mães internadas com suspeita ou diagnóstico do COVID-19 não devem extrair o leite materno no BLH. As demais podem realizar a ordenha no $B L H$, desde que respeitadas as normas de distanciamento e higiene padronizadas;

- A ordenha pode também ser realizada no apartamento. O profissional de enfermagem ou nutrição, devidamente paramentado (4 EPIs), entrega a bomba extratora à mãe e realiza as orientações;

- O leite coletado deve ser utilizado em até 12 horas ou ser pasteurizado e congelado no BLH, seguindo o fluxo normal de recomendação da Rede Nacional de $\mathrm{BLH}^{27}$;

- Após a retirada do frasco de leite coletado no apartamento, deve-se higienizar o frasco com álcool a $70 \%$, antes de levá-lo ao BLH ${ }^{27}$;

- A bomba extratora de leite materno deve ser recolhida em recipiente exclusivo e encaminhada para a área de higienização do lactário, para que seja higienizada conforme Procedimento Operacional Padrão (POP) aprovado pela $\mathrm{CClH}$ da instituição.

\section{CONCLUSÃo}

Não há um consenso a cerca de qual a melhor conduta a ser utilizada no tratamento de crianças e adolescentes com COVID-19. Temos sugerido a escolha da conduta que melhor se adeque à população atendida e à realidade de cada instituição com diferentes níveis de recursos. A terapia nutricional mantém-se como essencial para manter as funções vitais e a homeostase do organismo, recuperar a atividade do sistema imunológico e evitar a subnutrição.

\section{REFERÊNCIAS}

1. Cai J, Xu J, Lin D, Yang Z, Xu L, Qu Z, et al. A case series of children with 2019 novel coronavirus infection: clinical and epidemiological features. Clin Infect Dis. 2020;pii: ciaa198. doi: 10.1093/cid/ciaa198.

2. She J, Liu L, Liu W. COVID-19 epidemic: disease characteristics in children. J Med Virol. 2020;92(7):747-54.

3. Dong Y, Mo X, Hu Y, Qi X, Jiang F, Jiang Z, Tong S. Epidemiological characteristics of 2143 pediatric patients with 2019 coronavirus disease in China. Pediatrics. 2020; doi: 10.1542/ peds.2020-0702.

4. Lu X, Zhang L, Du H, Zhang J, Li YY, Qu J, et al. SARS-CoV-2 infection in children. N Engl J Med. 2020;382(17):1663-5.

5. Chen N, Zhou M, Dong X, Qu J, Gong F, Han Y, et al. Epidemiological and clinical characteristics of 99 cases of 2019 novel coronavirus pneumonia in Wuhan, China: a descriptive study. Lancet. 2020;395(10223):507-13.

6. CDC COVID-19 Response Team. Severe outcomes among patients with coronavirus disease 2019 (COVID-19) - United States, February 12-March 16, 2020. MMWR Morb Mortal Wkly Rep. 2020;69(12):343-6.

7. World Health Organization. Coronavirus disease (COVID19) pandemic. Geneva: World Health Organization; 2020. [cited 2020Mar 15].Disponívelem:https://www.who.int/emergencies/ diseases/novel-coronavirus-2019.

8. Hulst JM, Zwart H, Hop WC, Joosten KFM. Dutch national survey to test the STRONGkids nutritional risk screening tool in hospitalized children. Clin Nutr. 2010;29(1):106-11.

9. Brasil. Ministério da Saúde. Secretaria de Vigilância em Saúde. Centro de Operações de Emergência (COE) - Coronavírus. Portal da Agência Nacional de Vigilância Sanitária. Brasília: Ministério da Saúde; 2020. [cited 2020 Mar 15]. Disponível em: http://portal.anvisa.gov.br/coronavirus

10. Silva APA. Atuação do nutricionista em hospital pediátrico. In: Delgado AF, Cardoso AL, Zamberlan P, Tumas R, eds. Nutrologia. $2^{\mathrm{a}}$ ed. Barueri: Manole; 2019. p.88-105.

11. Associação Brasileira de Nutrição (ASBRAN). Manual orientativo: sistematização do cuidado de nutrição. São Paulo: Associação Brasileira de Nutrição; 2014.

12. Schofield WN. Predicting basal metabolic rate, new standards and review of previous work. Hum Nutr Clin Nutr. 1985;39(Suppl. 1):5-41

13. WorldHealth Organization. WHOMulticentre Growth Reference Study Group. WHO Child Growth Standards based on length/ height, weight and age. Acta Paediatr Suppl. 2006;450:76-85. 
14. Mehta NM, Skillman HE, Irving SY, Coss-Bu JA, Vermilyea S, Farrington EA, et al. Guidelines for the provision and assessment of nutrition support therapy in the pediatric critically ill patient: Society of Critical Care Medicine and American Society for Parenteral and Enteral Nutrition. JPEN J Parenter Enteral Nutr. 2017;41(5):706-42.

15. Gandolfo AS, Zamberlan P, Silva APA, Pinelli DPS, Feferbaum R. Algoritmos de nutrição enteral na pediatria. (Série de publicações ILSI Brasil: força-tarefa de nutrição da criança; v.6). São Paulo: ILSI Brasil-International Life Sciences Institute do Brasil; 2017

16. Brasil. Ministério da Saúde. Agência Nacional de Vigilância Sanitária, Portaria $n^{\circ} 272$, de 8 de abril de 1998. Aprova o regulamento técnico para fixar os requisitos mínimos exigidos para a terapia de nutrição parenteral. Brasília: Ministério da Saúde; 1998. [cited 2020 Mar 15]. Disponível em: http://bvsms.saude. gov.br/bvs/saudelegis/svs1/1998/prt0272_08_04_1998.html

17. Brasil. Ministério da Saúde. Agência Nacional de Vigilância Sanitária, RDC no 63, de 6 de julho de 2000. Aprova o regulamento técnico para a terapia de nutrição enteral. Brasília: Ministério da Saúde; 1999. [cited 2020 Mar 15]. Disponível em: http://bvsms.saude.gov.br/bvs/saudelegis/anvisa/2000/ rdc0063 $0607 \quad 2000 . h t m l$

18. Martindale R, Patel JJ, Taylor B, Arabi YM, Warren M, McClave SA. Nutrition therapy in critically ill patients with coronavirus disease (COVID-19). JPEN J Parenter Enteral Nutr. 2020;10.1002/jpen.1930. doi:10.1002/jpen.1930.

19. United States of America. Centers for Disease Control and Prevention (CDC). Interim considerations for infection prevention and control of coronavirus disease 2019 (COVID19) in inpatient obstetric healthcare settings. Atlanta: Centers for Disease Control and Prevention; 2020. [cited 2020 Mar 15]. Disponível em: https://www.cdc.gov/coronavirus/2019ncov/hcp/inpatient-obstetric-healthcare-guidance.html

20. Royal College of Obstetricians and Gynaecologists. Coronavirus (COVID-19) infection in pregnancy. Information for healthcare professionals. London: Royal College of Obstetricians and Gynaecologists; 2020. [cited 2020 Apr 15]. Disponível em: https://www.rcog.org.uk/globalassets/documents/ guidelines/2020-06-04-coronavirus-covid-19-infection-inpregnancy.pdf
21. Brasil. Ministério da Saúde. Fundação Oswaldo Cruz. Rede Brasileira de Bancos de Leite Humano. Recomendação Técnica No 03/20.160420. Assunto: Recomendações para acolhimento e manejo clínico em aleitamento materno de gestantes, puérperas e lactantes assintomáticas ou sintomáticas de COVID-19 pelo Banco de Leite Humano. Documento Brasília: Ministério da Saúde; 2020. [cited 2020 Mar 15]. Disponível em: https://rblh.fiocruz.br/ covid-19-recomendacao-tecnica-no0320160420

22. Brasil. Ministério da Saúde. Fundação Oswaldo Cruz. Rede Brasileira de Bancos de Leite Humano. COVID-19 e amamentação. Recomendação Técnica N.0120.170320. Brasília: Ministério da Saúde; 2020. [cited 2020 Mar 15]. Disponível em: https://rblh.fiocruz.br/ covid-19-e-amamentacao-recomendacao-n0120170320

23. Brasil. Ministério da Saúde. Cartilha para a mãe trabalhadora que amamenta. Brasília: Ministério da Saúde; 2020. [cited 2020 Mar 15]. Disponível em: http://bvsms.saude.gov.br/bvs/publicacoes/ cartilha_mulher_trabalhadora_amamenta.pdf

24. Galego DS, Ruotolo F, Barrios DW, Reis APA. Manual de boas práticas em nutrição enteral e lactário. Barueri: Manole; 2020.

25. Brasil. Secretaria Municipal de Saúde de São Paulo. Portaria $\mathrm{n}^{\mathrm{o}} 2619$, de 06 de dezembro de 2011. Regulamento de boas práticas e de controle de condições sanitárias e técnicas das atividades relacionadas à importação, exportação, extração, produção, manipulação, beneficiamento, acondicionamento, comercialização e uso de alimentos, águas minerais e de fontes, bebidas, aditivos e embalagens para alimentos. [cited $2020 \mathrm{Mar}$ 15]. Disponível em: http://www.prefeitura.sp.gov.br

26. Brasil. Ministério da Saúde. Agência Nacional de Vigilância Sanitária. Segurança do paciente em serviços de saúde: limpeza e desinfecção de superfícies. Brasília: Anvisa; 2012. p.118. [cited 2020 Mar 15]. Disponível em: https://www20. anvisa.gov.br/segurancadopaciente/index.php/publicacoes/ item/seguranca-do-paciente-em-servicos-de-saude-limpezae-desinfeccao-de-superficies

27. Brasil. Ministério da Saúde. Agência Nacional de Vigilância Sanitária. RDC n 171 , de 4 de setembro de 2006. Regulamento técnico para funcionamento de banco de leite humano. [cited 2020 Mar 15]. Disponível em: http://bvsms.saude.gov.br/bvs/ saudelegis/anvisa/2006/res0171_04_09_2006.html

Local de realização do estudo: Instituto da Criança e do Adolescente, Hospital das Clínicas, Faculdade de Medicina, Universidade de São Paulo, São Paulo, SP, Brasil.

Conflito de interesse: Os autores declaram não haver. 